\title{
Group Decision Making with Incomplete Interval-valued Fuzzy Preference Relations Based on the Minimum Operator
}

\author{
S. Ashraf, A. Rehman, E.E. Kerre
}

\author{
Samina Ashraf*, Atiq ur Rehman \\ Department of Mathematics \\ COMSATS Institute of Information Technology \\ Lahore, Pakistan \\ saminaa561@gmail.com, atiq.math@gmail.com \\ *Corresponding author: saminaa561@gmail.com \\ Etienne E. Kerre \\ Department of Applied Mathematics, \\ Computer Science, B-900 Gent, Belgium \\ etienne.kerre@UGent.be
}

\begin{abstract}
This paper presents a new method to estimate the unknown values in incomplete interval-valued fuzzy preference relations (IVFPRs). The method is based on the min-consistency and is used to develop the algorithm for group decision making (GDM) dealing with incomplete IVFPRs.

Keywords: fuzzy preference relation, interval-valued fuzzy preference relation; incomplete interval-valued fuzzy preference relation; min-consistency; group decision making.
\end{abstract}

\section{Introduction and preliminaries}

GDM is a situation faced when a number of experts work together to find the best alternative(s) from a set of feasible alternatives. Each expert may have exclusive inspirations or objectives and a different decision procedure, but has a common interest in approaching to select the "best" option(s). Preference relation is the most common representation format used in GDM because it is a valuable tool in modeling decision processes, when we have to combine experts' preferences into group preferences $[6,14,15]$. In a preference relation an expert assigns a numerical value to every pair of alternatives that reflects some degree of preference of the first alternative over the second alternative. Mainly two types of preference relations have been used to develop the decision models; multiplicative preference relations (MPRs) [2,14], and fuzzy preference relations (FPRs) $[6,16]$.

The popular preference relations, which are being used to express an expert's preferences over alternatives, are FPRs. In a decision making procedure, an expert mostly needs to compare a finite set of alternatives $x_{i}(i=1,2, \ldots, n)$ and construct an FPR $[6,13,16,17]$. However, an expert may have imprecise information for the preference degrees of one alternative over another and it may not always be possible to estimate his/her preference by means of an exact numerical value. In such a situation, an expert constructs an IVFPR.

In 2004, Z. S. Xu defined the notion of compatibility degree of two IVFPRs and showed the compatible connection among individual and collective IVFPRs [18]. In 2005, F. Herrera et al. established an aggregation process for combining IVFPRs with other forms of information as; numerical preference relation (NPR) and linguistic preference relation (LPR) [7]. In 2007, Y. Jiang proposed a technique to measure the similarity degree of two IVFPRs and used the error-propagation rule to find the priority vector of the accumulated IVFPRs [8]. In 2008, Z. $\mathrm{S}$. Xu and Chen developed some linear programming models to derive the priority weights from several IVFPRs [20]. 
All the above researches focused on the IVFPRs with complete information. However, in DM problems such situations are unavoidable in which an expert does not have comprehensive information of the problem because of time constraint, lack of knowledge and the expert's limited expertise within the problem domain $[1,3,5,10,19,22,24,33]$. Consequently, the expert may not be able to give his/her opinion about specific traits of the problem, and hence an incomplete preference relation would be constructed. In literature, researches based on incomplete FPRs have been given, but there are only few researches in GDM related to incomplete IVFPRs [23].

In this paper, a new technique for GDM by using incomplete IVFPRs is developed. Obviously, the consistent information is more applicable or important than the information having ambiguities, consistency is linked with definite transitivity properties. Several properties have been endorsed to model transitivity of FPRs, one of these properties is the max-min transitivity. In this paper, a procedure, based on min-transitivity property is proposed to determine unknown interval-valued preferences of one alternative over others and further, it is extended to develop an algorithm for GDM to select the best alternative.

Definition 1.1. [4] An interval-valued fuzzy set $A$ on a universe $X$ is defined as:

$$
A=\left\{\left(a,\left[x^{-}, x^{+}\right]\right) \mid a \in X,\left[x^{-}, x^{+}\right] \in L([0,1])\right\}
$$

where $L([0,1])=\left\{\left[x^{-}, x^{+}\right] \mid\left[x^{-}, x^{+}\right] \subseteq[0,1]\right.$ with $\left.x^{-} \leq x^{+}\right\}$.

Arithmetic operations can be performed on closed intervals. The following formulae can be used for all $P, Q \in L([0,1])\left(P=\left[p^{-}, p^{+}\right]\right.$and $\left.Q=\left[q^{-}, q^{+}\right]\right)[12]$ :

- $P+Q=\left[p^{-}+q^{-}, p^{+}+q^{+}\right]$,

- $P-Q=\left[p^{-}-q^{+}, p^{+}-q^{-}\right]$,

- $P \cdot Q=\left[\min \left(p^{-} q^{-}, p^{-} q^{+}, p^{+} q^{-}, p^{+} q^{+}\right), \max \left(p^{-} q^{-}, p^{-} q^{+}, p^{+} q^{-}, p^{+} q^{+}\right)\right]$,

- $P / Q=\left[p^{-}, p^{+}\right] \cdot\left[\frac{1}{q^{+}}, \frac{1}{q^{-}}\right]$if $0 \notin\left[q^{-}, q^{+}\right]$.

Definition 1.2. [4] Let $X$ be a universe and $A$ and $B$ two interval-valued fuzzy sets. The inclusion of $A$ into $B$ is defined as: $A \subseteq B$ if and only if $A(a) \subseteq B(a)$ for all $a \in X$ and the equality between $A$ and $B$ is defined as: $A=B$ if and only if $A(a)=B(a)$ for all $a \in X$.

Definition 1.3. [9] A triangular norm (t-norm) $T$ is an increasing, associative, commutative and $[0,1] \times[0,1] \rightarrow[0,1]$ mapping satisfying: $T(1, x)=x$ for all $x \in[0,1]$.

The t-norm to be used in this paper is $T(x, y)=\min (x, y)$. The concept of a t-norm on $[0,1]$ can be extended to subintervals of $[0,1]$.

Definition 1.4. An extended t-norm, $T_{e}$, is an increasing, commutative, associative and $L([0,1]) \times L([0,1]) \rightarrow L([0,1])$ mapping that satisfies:

$$
T_{e}\left([1,1],\left[x^{-}, x^{+}\right]\right)=\left[x^{-}, x^{+}\right] \text {for all }\left[x^{-}, x^{+}\right] \in L([0,1]) .
$$

Let $T$ be a triangular norm. The mapping $T_{e}$ defined as:

$$
T_{e}\left(\left[a^{-}, a^{+}\right],\left[b^{-}, b^{+}\right]\right)=\left[T\left(a^{-}, b^{-}\right), T\left(a^{+}, b^{+}\right)\right]
$$

for $\left[a^{-}, a^{+}\right],\left[b^{-}, b^{+}\right] \in L([0,1])$, is an extended t-norm on $(L([0,1]), \subseteq)$, where $\subseteq$ represents the crisp set inclusion.

The extended interval t-norm corresponding to the minimum-operator can be computed by:

$$
T_{\min }\left(\left[a^{-}, a^{+}\right],\left[b^{-}, b^{+}\right]\right)=\left[\min \left(a^{-}, b^{-}\right), \min \left(a^{+}, b^{+}\right)\right] .
$$


Definition 1.5. [15] A fuzzy preference relation $R$ over a finite set $X$ of alternatives, $X=$ $\left\{x_{1}, x_{2}, x_{3}, \ldots, x_{n}\right\}$, is a fuzzy set on the product set $X \times X$, i.e., it is characterized by a membership function $\mu_{R}: X \times X \rightarrow[0,1]$.

According to Definition 1.5, a fuzzy preference relation $R$ on $X$ can be conveniently expressed by an $n \times n$ matrix $R=\left(r_{i j}\right)_{n \times n}$, where $r_{i j}$ denotes the degree of preference of alternative $x_{i}$ over the alternative $x_{j}$ with $r_{i j} \in[0,1], r_{i i}=0.5, r_{i j}+r_{j i}=1$ (additive reciprocity) for $1 \leq i \leq n$ and $1 \leq j \leq n$. If $r_{i j}=0.5$, then there is no difference between the alternatives $x_{i}$ and $x_{j}$. If $r_{i j}>0.5$, then alternative $x_{i}$ is preferred over the alternative $x_{j}$. If $r_{i j}=1$, then the alternative $x_{i}$ is definitely preferred over the alternative $x_{j}$.

Definition 1.6. [18] Let $\bar{R}=\left(\bar{r}_{i j}\right)_{n \times n}$ be a fuzzy preference relation over the set of alternatives $X=\left\{x_{1}, x_{2}, x_{3}, \ldots, x_{n}\right\}$ where $\bar{r}_{i j}=\left[r_{i j}^{-}, r_{i j}^{+}\right], 0 \leq r_{i j}^{-} \leq r_{i j}^{+} \leq 1, \bar{r}_{i j}=[1,1]-\bar{r}_{j i}$ and $\bar{r}_{i i}=[0.5,0.5]$ for all $i, j \in N$, then $\bar{R}$ is called an interval-valued fuzzy preference relation.

Definition 1.7. An IVFPR $\bar{R}$ is said to be min-consistent, if for all $i, j$ and $k$ belonging to $\{1,2,3, \ldots, n\}$ it holds:

$$
\bar{r}_{i k} \geq T_{\min }\left(\bar{r}_{i j}, \bar{r}_{j k}\right) \quad \text { (min-transitivity) }
$$

Definition 1.8. An IVFPR relation $\bar{R}=\left(\bar{r}_{i j}\right)_{n \times n}$ is said to be incomplete if it contains at least one unknown preference value $\bar{r}_{i j}$ for which the expert has no idea about the degree of preference of alternative $x_{i}$ over the alternative $x_{j}$.

\section{Method to repair an incomplete IVFPR}

This section presents a new technique to estimate missing values in an incomplete IVFPR. Further, the algorithm is used to construct a min-consistent matrix. In order to determine unknown values in an incomplete IVFPR $\bar{R}=\left(\bar{r}_{i j}\right)_{n \times n}$, the pairs of alternatives for known and unknown preference values are represented by the following sets:

$$
\begin{aligned}
K_{P} & =\left\{(i, j) \mid \bar{r}_{i j} \text { is known }\right\}, \\
U_{P} & =\left\{(i, j) \mid \bar{r}_{i j} \text { is unknown }\right\},
\end{aligned}
$$

where the preference value of alternative $x_{i}$ over $x_{j}$ belongs to the family of closed subintervals of $[0,1]$ (i.e., $\bar{r}_{i j} \in L([0,1])$ ). Since $\bar{r}_{i j}=[1,1]-\bar{r}_{j i}, \bar{r}_{i i}=[0.5,0.5]$ for $1 \leq i \leq n$ and $1 \leq j \leq n$, therefore, the min-transitivity of definition 1.7 can be written as:

$$
\bar{r}_{i k} \geq T_{\min }\left(\bar{r}_{i j}, \bar{r}_{j k}\right) ; \bar{r}_{i k} \geq T_{\min }\left(1-\bar{r}_{j i}, \bar{r}_{j k}\right) ; \bar{r}_{i k} \geq T_{\min }\left(\bar{r}_{i j}, 1-\bar{r}_{k j}\right) .
$$

Hence, the following sets can be defined to determine the unknown preference value $\bar{r}_{i k}$ of alternative $x_{i}$ over alternative $x_{k}$ :

$$
\begin{aligned}
& S_{i k}^{1}=\left\{j \mid(i, j) \in K_{P},(j, k) \in K_{P} \text { and }(i, k) \in U_{P}\right\}, \\
& S_{i k}^{2}=\left\{j \mid(j, i) \in K_{P},(j, k) \in K_{P} \text { and }(i, k) \in U_{P}\right\}, \\
& S_{i k}^{3}=\left\{j \mid(i, j) \in K_{P},(k, j) \in K_{P} \text { and }(i, k) \in U_{P}\right\},
\end{aligned}
$$

for $i=\{1,2,3, \ldots, n\}, j=\{1,2,3, \ldots, n\}$ and $k=\{1,2,3, \ldots, n\}$. Based on (5),(6) and (7), we can determine the unknown preference value $\bar{r}_{i k}$ for $x_{i}$ over $x_{k}$ as follows:

$$
\bar{r}_{i k}=\frac{\bar{r}_{i k}^{1}+\bar{r}_{i k}^{2}+\bar{r}_{i k}^{3}}{3},
$$


where

$$
\begin{aligned}
& \bar{r}_{i k}^{1}= \begin{cases}\frac{1}{\left|S_{i k}^{1}\right|} \sum_{j \in S_{i k}^{1}} T_{\min }\left(\bar{r}_{i j}, \bar{r}_{j k}\right), & \text { if }\left|S_{i k}^{1}\right| \neq 0 \\
{[0.5,0.5],} & \text { otherwise }\end{cases} \\
& \bar{r}_{i k}^{2}= \begin{cases}\frac{1}{\left|S_{i k}^{2}\right|} \sum_{j \in S_{i k}^{2}} T_{\min }\left([1,1]-\bar{r}_{j i}, \bar{r}_{j k}\right), & \text { if }\left|S_{i k}^{2}\right| \neq 0 \\
{[0.5,0.5],} & \text { otherwise }\end{cases} \\
& \bar{r}_{i k}^{3}= \begin{cases}\frac{1}{\left|S_{i k}^{3}\right|} \sum_{j \in S_{i k}^{3}} T_{\min }\left(\bar{r}_{i j},[1,1]-\bar{r}_{k j}\right), & \text { if }\left|S_{i k}^{3}\right| \neq 0 \\
{[0.5,0.5],} & \text { otherwise }\end{cases}
\end{aligned}
$$

where $\left|S_{i k}^{1}\right|,\left|S_{i k}^{2}\right|$ and $\left|S_{i k}^{3}\right|$ are the cardinalities of the sets $S_{i k}^{1}, S_{i k}^{2}$ and $S_{i k}^{3}$ respectively.

$$
\begin{aligned}
K_{P}^{\prime} & =K_{P} \cup\{(i, k)\}, \\
U_{P}^{\prime} & =U_{P}-\{(i, k)\} .
\end{aligned}
$$

To achieve min-consistency of the IVFPR $\bar{R}$, following scaling conditions will be used:

(i) If $r_{i j}^{-}+r_{j i}^{+}<1$ and $r_{i j}^{+}+r_{j i}^{-}<1$, then

$$
\bar{r}_{i j}=\left[r_{i j}^{-}+\frac{1-\left(r_{i j}^{-}+r_{j i}^{+}\right)}{2}, r_{i j}^{+}+\frac{1-\left(r_{i j}^{+}+r_{j i}^{-}\right)}{2}\right]
$$

and

$$
\bar{r}_{j i}=\left[r_{j i}^{-}+\frac{1-\left(r_{i j}^{+}+r_{j i}^{-}\right)}{2}, r_{j i}^{+}+\frac{1-\left(r_{i j}^{-}+r_{j i}^{+}\right)}{2}\right] .
$$

(ii) If $r_{i j}^{-}+r_{j i}^{+}<1$ and $r_{i j}^{+}+r_{j i}^{-}>1$, then

$$
\bar{r}_{i j}=\left[r_{i j}^{-}+\frac{1-\left(r_{i j}^{-}+r_{j i}^{+}\right)}{2}, r_{i j}^{+}-\frac{r_{i j}^{+}+r_{j i}^{-}-1}{2}\right]
$$

and

$$
\bar{r}_{j i}=\left[r_{j i}^{-}-\frac{r_{i j}^{+}+r_{j i}^{-}-1}{2}, r_{j i}^{+}+\frac{1-\left(r_{i j}^{-}+r_{j i}^{+}\right)}{2}\right] .
$$

(iii) If $r_{i j}^{-}+r_{j i}^{+}>1$ and $r_{i j}^{+}+r_{j i}^{-}<1$, then

$$
\bar{r}_{i j}=\left[r_{i j}^{-}-\frac{r_{i j}^{-}+r_{j i}^{+}-1}{2}, r_{i j}^{+}+\frac{1-\left(r_{i j}^{+}+r_{j i}^{-}\right)}{2}\right]
$$

and

$$
\bar{r}_{j i}=\left[r_{j i}^{-}+\frac{1-\left(r_{i j}^{+}+r_{j i}^{-}\right)}{2}, r_{j i}^{+}-\frac{r_{i j}^{-}+r_{j i}^{+}-1}{2}\right] .
$$

(iv) If $r_{i j}^{-}+r_{j i}^{+}>1$ and $r_{i j}^{+}+r_{j i}^{-}>1$, then

$$
\bar{r}_{i j}=\left[r_{i j}^{-}-\frac{r_{i j}^{-}+r_{j i}^{+}-1}{2}, r_{i j}^{+}-\frac{r_{i j}^{+}+r_{j i}^{-}-1}{2}\right]
$$


and

$$
\bar{r}_{j i}=\left[r_{j i}^{-}-\frac{r_{i j}^{+}+r_{j i}^{-}-1}{2}, r_{j i}^{+}-\frac{r_{i j}^{-}+r_{j i}^{+}-1}{2}\right] .
$$

Example 2.1. Let $\bar{R}=\left(\bar{r}_{i j}\right)_{4 \times 4}$ be an incomplete IVFPR for the alternatives $x_{1}, x_{2}, x_{3}$ and $x_{4}$, given as follows:

$$
\bar{R}=\left[\begin{array}{cccc}
{[0.5,0.5]} & \bar{r}_{12} & {[0.4,0.6]} & {[0.3,0.7]} \\
\bar{r}_{21} & {[0.5,0.5]} & {[0.7,0.8]} & \bar{r}_{24} \\
{[0.4,0.6]} & {[0.2,0.3]} & {[0.5,0.5]} & {[0.3,0.4]} \\
{[0.3,0.7]} & \bar{r}_{42} & {[0.6,0.7]} & {[0.5,0.5]}
\end{array}\right]
$$

where $\bar{r}_{12}, \bar{r}_{21}, \bar{r}_{24}$ and $\bar{r}_{42}$ are unknown preference values. Now applying (2)-(13) to estimate the unknown preference values for the alternative $x_{i}$ over $x_{k}, 1 \leq i \leq 4$ and $1 \leq k \leq 4$, we obtain:

$$
\begin{aligned}
K_{P}= & \{(1,1),(1,3),(1,4),(2,2),(2,3),(3,1),(3,2),(3,3),(3,4),(4,1), \\
& (4,3),(4,4)\}, \\
U_{P}= & \{(1,2),(2,1),(2,4),(4,2)\} . \\
S_{12}^{1}= & \{3\}, \quad S_{12}^{2}=\{3\}, \quad S_{12}^{3}=\{3\}, \\
\bar{r}_{12}^{1}= & T_{\min }\left(\bar{r}_{13}, \bar{r}_{32}\right)=T_{\min }([0.4,0.6],[0.2,0.3])=[0.2,0.3], \\
\bar{r}_{12}^{2}= & T_{\min }\left([1,1]-\bar{r}_{31}, \bar{r}_{32}\right)=T_{\min }([0.4,0.6],[0.2,0.3])=[0.2,0.3], \\
\bar{r}_{12}^{3}= & T_{\min }\left(\bar{r}_{13},[1,1]-\bar{r}_{23}\right)=T_{\min }([0.4,0.6],[0.2,0.3])=[0.2,0.3], \\
\bar{r}_{12}= & \frac{1}{3}\left(\bar{r}_{12}^{1}+\bar{r}_{12}^{2}+\bar{r}_{12}^{3}\right)=[0.2,0.3] . \\
K_{P}^{\prime}= & \{(1,1),(1,2),(1,3),(1,4),(2,2),(2,3),(3,1),(3,2),(3,3),(3,4), \\
& (4,1),(4,3),(4,4)\}, \\
U_{P}^{\prime}= & U_{P}-\{(1,2)\}=\{(2,1),(2,4),(4,2)\} . \\
S_{21}^{1}= & \{3\}, \quad S_{21}^{2}=\{1,3\}, \quad S_{21}^{3}=\{2,3\}, \\
\bar{r}_{21}^{1}= & T_{\min }\left(\bar{r}_{23}, \bar{r}_{31}\right)=T_{\min }([0.7,0.8],[0.4,0.6])=[0.4,0.6], \\
\bar{r}_{21}^{2}= & \frac{1}{2}\left[T_{\min }\left([1,1]-\bar{r}_{12}, \bar{r}_{11}\right)+T_{\min }\left([1,1]-\bar{r}_{32}, \bar{r}_{31}\right)\right] \\
= & \frac{1}{2}\left[T_{\min }([0.7,0.8],[0.5,0.5])+T_{\min }([0.7,0.8],[0.4,0.6])\right] \\
= & \frac{1}{2}[[0.5,0.5]+[0.4,0.6]]=[0.45,0.55],
\end{aligned}
$$




$$
\begin{aligned}
& \bar{r}_{21}^{3}=\frac{1}{2}\left[T_{\min }\left(\bar{r}_{22},[1,1]-\bar{r}_{12}\right)+T_{\min }\left(\bar{r}_{23},[1,1]-\bar{r}_{13}\right)\right] \\
& =\frac{1}{2}\left[T_{\min }([0.5,0.5],[0.7,0.8])+T_{\min }([0.7,0.8],[0.4,0.6]]\right. \\
& =\frac{1}{2}[[0.5,0.5]+[0.4,0.6]]=[0.45,0.55] \text {, } \\
& \bar{r}_{21}=\frac{1}{3}\left(\bar{r}_{21}^{1}+\bar{r}_{21}^{2}+\bar{r}_{21}^{3}\right)=[0.433,0.567] \text {. } \\
& K_{P}^{\prime \prime}=\{(1,1),(1,2),(1,3),(1,4),(2,1),(2,2),(2,3),(3,1),(3,2),(3,3),(3,4), \\
& (4,1),(4,3),(4,4)\} \text {, } \\
& U_{P}^{\prime \prime}=U^{\prime}-\{(2,1)\}=\{(2,4),(4,2)\} . \\
& S_{24}^{1}=\{1,3\}, \quad S_{24}^{2}=\{1,3\}, \quad S_{24}^{3}=\{1,3\}, \\
& \bar{r}_{24}^{1}=\frac{1}{2}\left[T_{\min }\left(\bar{r}_{21}, \bar{r}_{14}\right)+T_{\min }\left(\bar{r}_{23}, \bar{r}_{34}\right)\right] \\
& =\frac{1}{2}\left[T_{\min }([0.433,0.567],[0.3,0.7])+T_{\min }([0.7,0.8],[0.3,0.4])\right] \\
& =\frac{1}{2}[[0.3,0.567]+[0.3,0.4]]=[0.3,0.484] \text {, } \\
& \bar{r}_{24}^{2}=\frac{1}{2}\left[T_{\min }\left([1,1]-\bar{r}_{12}, \bar{r}_{14}\right)+T_{\min }\left([1,1]-\bar{r}_{32}, \bar{r}_{34}\right)\right] \\
& =\frac{1}{2}\left[T_{\min }([0.7,0.8],[0.3,0.7])+T_{\min }([0.7,0.8],[0.3,0.4])\right] \\
& =\frac{1}{2}[[0.3,0.7]+[0.3,0.4]]=[0.3,0.55], \\
& \bar{r}_{24}^{3}=\frac{1}{2}\left[T_{\min }\left(\bar{r}_{21},[1,1]-\bar{r}_{41}\right)+T_{\min }\left(\bar{r}_{23},[1,1]-\bar{r}_{43}\right)\right] \\
& =\frac{1}{2}\left[T_{\min }([0.433,0.567],[0.3,0.7])+T_{\min }([0.7,0.8],[0.3,0.4])\right] \\
& =\frac{1}{2}[[0.3,0.567]+[0.3,0.4]]=[0.3,0.484] \text {, } \\
& \bar{r}_{24}=\frac{1}{3}\left(\bar{r}_{24}^{1}+\bar{r}_{24}^{2}+\bar{r}_{24}^{3}\right)=[0.3,0.506] . \\
& K_{P}^{\prime \prime \prime}=\{(1,1),(1,2),(1,3),(1,4),(2,1),(2,2),(2,3),(2,4),(3,1),(3,2),(3,3), \\
& (3,4),(4,1),(4,3),(4,4)\} \text {, } \\
& U_{P}^{\prime \prime \prime}=U^{\prime \prime}-\{(2,4)\}=\{(4,2)\} \text {. } \\
& S_{42}^{1}=\{1,3\}, \quad S_{42}^{2}=\{1,2,3\}, \quad S_{42}^{3}=\{1,3,4\}, \\
& \bar{r}_{42}^{1}=\frac{1}{2}\left[T_{\min }\left(\bar{r}_{41}, \bar{r}_{12}\right)+T_{\min }\left(\bar{r}_{43}, \bar{r}_{32}\right)\right] \\
& =\frac{1}{2}\left[T_{\min }([0.3,0.7],[0.2,0.3])+T_{\min }([0.6,0.7],[0.2,0.3])\right] \\
& =\frac{1}{2}[[0.2,0.3]+[0.2,0.3]]=[0.2,0.3] \text {, } \\
& \bar{r}_{42}^{2}=\frac{1}{3}\left[T_{\min }\left([1,1]-\bar{r}_{14}, \bar{r}_{12}\right)+T_{\min }\left([1,1]-\bar{r}_{24}, \bar{r}_{22}\right)+T_{\min }\left([1,1]-\bar{r}_{34}, \bar{r}_{32}\right)\right] \\
& =\frac{1}{3}[[0.2,0.3]+[0.5,0.494]+[0.2,0.3]] \\
& =[0.298,0.367] \text {, }
\end{aligned}
$$




$$
\begin{aligned}
\bar{r}_{42}^{3}= & \frac{1}{3}\left[T_{\min }\left(\bar{r}_{41},[1,1]-\bar{r}_{21}\right)+T_{\min }\left(\bar{r}_{43},[1,1]-\bar{r}_{23}\right)+T_{\min }\left(\bar{r}_{44},[1,1]-\bar{r}_{24}\right)\right] \\
= & \frac{1}{3}[[0.3,0.567]+[0.2,0.3]+[0.494,0.5]] \\
= & {[0.331,0.456], } \\
\bar{r}_{42}= & \frac{1}{3}\left(\bar{r}_{42}^{1}+\bar{r}_{42}^{2}+\bar{r}_{42}^{3}\right)=[0.276,0.374] . \\
K_{P}^{\prime \prime \prime \prime \prime}= & \{(1,1),(1,2),(1,3),(1,4),(2,1),(2,2),(2,3),(2,4),(3,1),(3,2),(3,3), \\
& (3,4),(4,1),(4,2),(4,3),(4,4)\}, \\
U_{P}^{\prime \prime \prime \prime}= & \phi .
\end{aligned}
$$

Hence, the complete IVFPR is

$$
\bar{R}=\left[\begin{array}{cccc}
{[0.5,0.5]} & {[0.2,0.3]} & {[0.4,0.6]} & {[0.3,0.7]} \\
{[0.433,0.567]} & {[0.5,0.5]} & {[0.7,0.8]} & {[0.3,0.506]} \\
{[0.4,0.6]} & {[0.2,0.3]} & {[0.5,0.5]} & {[0.3,0.4]} \\
{[0.3,0.7]} & {[0.276,0.374]} & {[0.6,0.7]} & {[0.5,0.5]}
\end{array}\right]
$$

By applying scaling condition on (18), $\bar{R}$ becomes a min-consistent IVFPR $\widetilde{\bar{R}}$ as follows:

$$
\widetilde{\bar{R}}=\left[\begin{array}{cccc}
{[0.5,0.5]} & {[0.316,0.434]} & {[0.4,0.6]} & {[0.3,0.7]} \\
{[0.566,0.684]} & {[0.5,0.5]} & {[0.7,0.8]} & {[0.463,0.615]} \\
{[0.4,0.6]} & {[0.2,0.3]} & {[0.5,0.5]} & {[0.3,0.4]} \\
{[0.3,0.7]} & {[0.385,0.537]} & {[0.6,0.7]} & {[0.5,0.5]}
\end{array}\right]
$$

\section{A new algorithm to choose the best alternative in GDM with incomplete IVFPRs.}

In this section, a new algorithm is presented for GDM with incomplete IVFPRs by using min-consistency. An explanatory example is given to validate the anticipated technique. For ease, the structure of the determination process is also shown in Figure1. Suppose that there are $n$ alternatives $x_{1}, x_{2}, \ldots, x_{n}$ and $m$ experts $E_{1}, E_{2}, \ldots, E_{m}$. Let $\bar{R}^{q}$ be the IVFPR for the expert $E_{q}$ shown as follows:

$$
\bar{R}^{q}=\left(\bar{r}_{i j}^{q}\right)_{n \times n}=\left[\begin{array}{ccccc}
{[0.5,0.5]} & \bar{r}_{12}^{q} & . & . & \bar{r}_{1 n}^{q} \\
\bar{r}_{21}^{q} & {[0.5,0.5]} & . & . & \bar{r}_{2 n}^{q} \\
\cdot & \cdot & \cdot & \cdot \\
\cdot & \cdot & & \cdot & \cdot \\
\bar{r}_{n 1}^{q} & \bar{r}_{n 2}^{q} & . & \cdot & {[0.5,0.5]}
\end{array}\right],
$$

where $\bar{r}_{i j}^{q} \in L([0,1])$ is the preference value given by expert $E_{q}$ for alternative $x_{i}$ over $x_{j}, \bar{r}_{i j}^{q}=$ $[1,1]-\bar{r}_{j i}^{q}, \bar{r}_{i i}^{q}=[0.5,0.5], 1 \leq i \leq n, 1 \leq j \leq n$ and $1 \leq q \leq m$. The proposed GDM technique is given as follows:

Step 1: Determine the sets $K_{P}^{q}$ and $U_{P}^{q}$ of pairs of alternatives for known and unknown preference values respectively, shown as follows:

$$
\begin{aligned}
K_{P}^{q} & =\left\{(i, j) \mid \bar{r}_{i j}^{q} \text { is known }\right\}, \\
U_{P}^{q} & =\left\{(i, j) \mid \bar{r}_{i j}^{q} \text { is unknown }\right\},
\end{aligned}
$$


where $1 \leq i \leq n, 1 \leq j \leq n$ and $1 \leq q \leq m$.

Step 2: If $U=\phi$, then skip Step 2, otherwise construct the sets $S_{i k}^{q 1}, S_{i k}^{q 2}$ and $S_{i k}^{q 3}$ based on the sets $K_{P}^{q}$ and $U_{P}^{q}$. The constructed sets are used to estimate the unknown preference values $\bar{r}_{i k}^{q}$ for the alternative $x_{i}$ over $x_{k}$ by expert $E_{q}$ as follows:

$$
\begin{aligned}
& \bar{r}_{i k}=\frac{\bar{r}_{i k}^{q 1}+\bar{r}_{i k}^{q 2}+\bar{r}_{i k}^{q 3}}{3}, \\
& S_{i k}^{q 1}=\left\{j \mid(i, j) \in K_{P}^{q},(j, k) \in K_{P}^{q} \text { and }(i, k) \in U_{P}^{q}\right\}, \\
& S_{i k}^{q 2}=\left\{j \mid(j, i) \in K_{P}^{q},(j, k) \in K_{P}^{q} \text { and }(i, k) \in U_{P}^{q}\right\} \text {, } \\
& S_{i k}^{q 3}=\left\{j \mid(i, j) \in K_{P}^{q},(k, j) \in K_{P}^{q} \text { and }(i, k) \in U_{P}^{q}\right\} \text {, } \\
& \bar{r}_{i k}^{q 1}=\left\{\begin{array}{lc}
\frac{1}{\left|S_{i k}^{q 1}\right|} \sum_{j \in S_{i k}^{q 1}} T_{\min }\left(\bar{r}_{i j}^{q}, \bar{r}_{j k}^{q}\right), & \text { if }\left|S_{i k}^{q 1}\right| \neq 0 \\
{[0.5,0.5],} & \text { otherwise }
\end{array}\right. \\
& \bar{r}_{i k}^{q 2}= \begin{cases}\frac{1}{\left|S_{i k}^{q 2}\right|} \sum_{j \in S_{i k}^{q 2}} T_{\min }\left([1,1]-\bar{r}_{i j}^{q}, \bar{r}_{j k}^{q}\right), & \text { if }\left|S_{i k}^{q 2}\right| \neq 0 \\
{[0.5,0.5],} & \text { otherwise }\end{cases} \\
& \bar{r}_{i k}^{q 3}=\left\{\begin{array}{lr}
\frac{1}{\left|S_{i k}^{q 3}\right|} \sum_{j \in S_{i k}^{q 3}} T_{\min }\left(\bar{r}_{i j}^{q},[1,1]-\bar{r}_{j k}^{q}\right), & \text { if }\left|S_{i k}^{q 3}\right| \neq 0 \\
{[0.5,0.5],} & \text { otherwise }
\end{array}\right.
\end{aligned}
$$

where $\left|S_{i k}^{q 1}\right|,\left|S_{i k}^{q 2}\right|$ and $\left|S_{i k}^{q 3}\right|$ are the cardinalities of the sets $S_{i k}^{q 1}, S_{i k}^{q 2}$ and $S_{i k}^{q 3}$ respectively.

$$
\begin{aligned}
K_{P}^{\prime q} & =K_{P}^{q} \cup\{(i, k)\}, \\
U_{P}^{\prime q} & =U_{P}^{q}-\{(i, k)\} .
\end{aligned}
$$

Step 3: To satisfy min-consistency of the complete interval-valued fuzzy preference relation $\bar{R}^{q}=\left(\bar{r}_{i j}^{q}\right)_{n \times n}$, the following scaling conditions are used:

(i) If $r_{i j}^{q-}+r_{j i}^{q+}<1$ and $r_{i j}^{q+}+r_{j i}^{q-}<1$, then

$$
\bar{r}_{i j}^{q}=\left[r_{i j}^{q-}+\frac{1-\left(r_{i j}^{q-}+r_{j i}^{q+}\right)}{2}, r_{i j}^{q+}+\frac{1-\left(r_{i j}^{q+}+r_{j i}^{q-}\right)}{2}\right]
$$

and

$$
\bar{r}_{j i}^{q}=\left[r_{j i}^{q-}+\frac{1-\left(r_{i j}^{q+}+r_{j i}^{q-}\right)}{2}, r_{j i}^{q+}+\frac{1-\left(r_{i j}^{q-}+r_{j i}^{q+}\right)}{2}\right] .
$$

(ii) If $r_{i j}^{q-}+r_{j i}^{q+}<1$ and $r_{i j}^{q+}+r_{j i}^{q-}>1$, then

$$
\bar{r}_{i j}^{q}=\left[r_{i j}^{q-}+\frac{1-\left(r_{i j}^{q-}+r_{j i}^{q+}\right)}{2}, r_{i j}^{q+}-\frac{r_{i j}^{q+}+r_{j i}^{q-}-1}{2}\right]
$$

and

$$
\bar{r}_{j i}^{q}=\left[r_{j i}^{q-}-\frac{r_{i j}^{q+}+r_{j i}^{q-}-1}{2}, r_{j i}^{q+}+\frac{1-\left(r_{i j}^{q-}+r_{j i}^{q+}\right)}{2}\right] .
$$


(iii) If $r_{i j}^{q-}+r_{j i}^{q+}>1$ and $r_{i j}^{q+}+r_{j i}^{q-}<1$, then

$$
\bar{r}_{i j}^{q}=\left[r_{i j}^{q-}-\frac{r_{i j}^{q-}+r_{j i}^{q+}-1}{2}, r_{i j}^{q+}+\frac{1-\left(r_{i j}^{q+}+r_{j i}^{q-}\right)}{2}\right]
$$

and

$$
\bar{r}_{j i}^{q}=\left[r_{j i}^{q-}+\frac{1-\left(r_{i j}^{q+}+r_{j i}^{q-}\right)}{2}, r_{j i}^{q+}-\frac{r_{i j}^{q-}+r_{j i}^{q+}-1}{2}\right] .
$$

(iv) If $r_{i j}^{q-}+r_{j i}^{q+}>1$ and $r_{i j}^{q+}+r_{j i}^{q-}>1$, then

$$
\bar{r}_{i j}^{q}=\left[r_{i j}^{q-}-\frac{r_{i j}^{q-}+r_{j i}^{q+}-1}{2}, r_{i j}^{q+}-\frac{r_{i j}^{q+}+r_{j i}^{q-}-1}{2}\right]
$$

and

$$
\bar{r}_{j i}^{q}=\left[r_{j i}^{q-}-\frac{r_{i j}^{q+}+r_{j i}^{q-}-1}{2}, r_{j i}^{q+}-\frac{r_{i j}^{q-}+r_{j i}^{q+}-1}{2}\right] .
$$

A min-consistent matrix $\widetilde{\bar{R}}^{q}=\left(\widetilde{\bar{r}}_{i j}^{q}\right)_{n \times n}$ is obtained under these conditions.

Step 4: Determine the collective matrix $\bar{R}^{c}$ against all experts, shown as follows:

$$
\bar{R}^{c}=\left(\bar{r}_{i j}^{c}\right)_{n \times n}=\frac{1}{m}\left(\widetilde{\bar{r}}_{i j}^{1}+\widetilde{\bar{r}}_{i j}^{2}+\widetilde{\bar{r}}_{i j}^{3} \ldots+\widetilde{\bar{r}}_{i j}^{m}\right)_{n \times n},
$$

where $1 \leq i \leq n, 1 \leq j \leq n$.

Step 5: Calculate the average degree $\bar{A}_{i}$ of alternative $x_{i}$ over all other alternatives by using interval normalizing method:

$$
\bar{A}_{i}=\frac{\sum_{j=1}^{n} \bar{r}_{i j}^{c}}{\sum_{i=1}^{n} \sum_{j=1}^{n} \bar{r}_{i j}^{c}}, \quad i=1,2,3, \ldots, n
$$

Step 6: [21] Calculate the possibility degree $d_{i j}=d\left(\bar{A}_{i} \geq \bar{A}_{j}\right)$ by using the formula:

$$
d\left(\bar{A}_{i} \geq \bar{A}_{j}\right)=\min \left\{\max \left(\frac{A_{i}^{+}-A_{j}^{-}}{A_{i}^{+}-A_{j}^{-}+A_{j}^{+}-A_{i}^{-}}, 0\right), 1\right\}
$$

and construct the complementry matrix $D=\left(d_{i j}\right)_{n \times n}$, where $d_{i j} \geq 0, d_{i j}+d_{j i}=1, d_{i i}=0$, $i, j=1,2,3, \ldots, n$.

Step 7: [33] Calculate the ranking value $R_{V}\left(x_{i}\right)$ of alternative $x_{i}$ by using formula:

$$
R_{V}\left(x_{i}\right)=\frac{2}{n^{2}} \sum_{j=1}^{n} d_{i j}
$$

where $1 \leq i \leq n$ and $\sum_{i=1}^{n} R_{V}\left(x_{i}\right)=1$. 


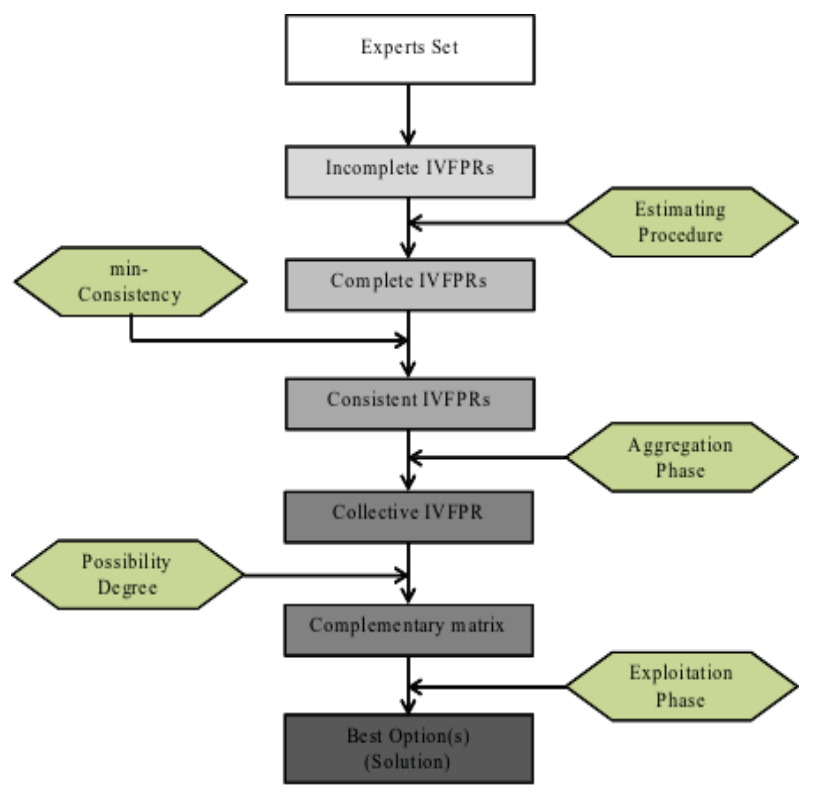

Figure 1: Resolution process for GDM with IVFPRs.

Example 3.1. A firm produces solar water refiners. In its production process, the company has to buy solar panels in different sizes and voltages from different suppliers. Presently, Japan Solar Company has four potential suppliers in four different countries, namely, Korea, China, Italy and Turkey, signified as $x_{i}(i=1,2,3,4)$, respectively. A committee consisting of three experts $E_{q}(q=1,2,3)$ from different departments has been formed to assess the four suppliers $x_{i}(i=1,2,3,4)$. Suppose that the experts $E_{q}(q=1,2,3)$ provide their assessments in the form of following incomplete IVFPRs:

and

$$
\begin{gathered}
\bar{R}^{1}=\left[\begin{array}{cccc}
{[0.5,0.5]} & \bar{r}_{12}^{1} & {[0.6,0.8]} & \bar{r}_{14}^{1} \\
\bar{r}_{21}^{1} & {[0.5,0.5]} & \bar{r}_{23}^{1} & {[0.3,0.7]} \\
{[0.2,0.4]} & \bar{r}_{32}^{1} & {[0.5,0.5]} & {[0.6,0.9]} \\
\bar{r}_{41}^{1} & {[0.3,0.7]} & {[0.1,0.4]} & {[0.5,0.5]}
\end{array}\right], \\
\bar{R}^{2}=\left[\begin{array}{cccc}
{[0.5,0.5]} & \bar{r}_{12} & {[0.4,0.6]} & {[0.3,0.7]} \\
\bar{r}_{21} & {[0.5,0.5]} & {[0.7,0.8]} & \bar{r}_{24} \\
{[0.4,0.6]} & {[0.2,0.3]} & {[0.5,0.5]} & {[0.3,0.4]} \\
{[0.3,0.7]} & \bar{r}_{42} & {[0.6,0.7]} & {[0.5,0.5]}
\end{array}\right],
\end{gathered}
$$

$$
\bar{R}^{3}=\left[\begin{array}{cccc}
{[0.5,0.5]} & \bar{r}_{12}^{3} & {[0.7,0.8]} & \bar{r}_{14}^{3} \\
{[0.4,0.6]} & {[0.5,0.5]} & \bar{r}_{23}^{3} & {[0.5,0.7]} \\
{[0.2,0.3]} & \bar{r}_{32}^{3} & {[0.5,0.5]} & \bar{r}_{34}^{3} \\
\bar{r}_{41}^{3} & {[0.3,0.5]} & \bar{r}_{43}^{3} & {[0.5,0.5]}
\end{array}\right] .
$$

Step 1: For the fuzzy preference relation $\bar{R}^{1}$, the sets of pairs of alternatives for known and unknown preference values are determined as follows:

$$
\begin{aligned}
K_{P}^{1} & =\{(1,1),(1,3),(2,2),(2,4),(3,1),(3,3),(3,4),(4,2),(4,3),(4,4)\} \\
U_{P}^{1} & =\{(1,2),(1,4),(2,1),(2,3),(3,2),(4,1)\} .
\end{aligned}
$$


Step 2:

$$
\begin{aligned}
S_{12}^{11}= & \phi, \quad S_{12}^{12}=\phi, \quad S_{12}^{13}=\phi, \\
\bar{r}_{12}^{11}= & {[0.5,0.5], \quad \bar{r}_{12}^{12}=[0.5,0.5], \quad \bar{r}_{12}^{13}=[0.5,0.5], } \\
\bar{r}_{12}^{1}= & \frac{1}{3}\left(\bar{r}_{12}^{11}+\bar{r}_{12}^{12}+\bar{r}_{12}^{13}\right) \\
= & {[0.5,0.5] . } \\
K_{P}^{1^{\prime}}= & \{(1,1),(1,2),(1,3),(2,2),(2,4),(3,1),(3,3),(3,4),(4,2),(4,3), \\
& (4,4)\}, \\
U_{P}^{1^{\prime}}= & U_{P}^{1}-\{(1,2)\}=\{(1,4),(2,1),(2,3),(3,2),(4,1)\} . \\
S_{14}^{11}= & \{2,3\}, \quad S_{14}^{12}=\{3\}, \quad S_{14}^{13}=\{2,3\}, \\
\bar{r}_{14}^{11}= & \frac{1}{2}\left[T_{\min }\left(\bar{r}_{12}^{1}, \bar{r}_{24}^{1}\right)+T_{\min }\left(\bar{r}_{13}^{1}, \bar{r}_{34}^{1}\right)\right]=\frac{1}{2}[[0.3,0.5]+[0.6,0.8]] \\
= & {[0.45,0.65], } \\
\bar{r}_{14}^{12}= & T_{\min }\left([1,1]-\bar{r}_{13}^{1}, \bar{r}_{34}^{1}\right)=T_{\min }([0.2,0,4],[0.6,0.9])=[0.2,0.4], \\
\bar{r}_{14}^{13}= & \frac{1}{2}\left[T_{\min }\left(\bar{r}_{12}^{1},[1,1]-\bar{r}_{42}^{1}\right)+T_{\min }\left(\bar{r}_{13}^{1},[1,1]-\bar{r}_{43}^{1}\right)\right] \\
= & \frac{1}{2}\left[T_{\min }([0.5,0.5],[0.3,0.7])+T_{\min }([0.6,0.8],[0.6,0.9])\right] \\
= & \frac{1}{2}[[0.3,0.5]+[0.6,0.8]]=[0.45,0.65], \\
\bar{r}_{14}^{1}= & \frac{1}{3}\left(\bar{r}_{14}^{11}+\bar{r}_{14}^{12}+\bar{r}_{14}^{13}\right)=[0.3667,0.5667] . \\
K_{P}^{1^{\prime \prime}}= & \{(1,1),(1,2),(1,3),(1,4),(2,2),(2,4),(3,1),(3,3),(3,4),(4,2), \\
& (4,3),(4,4)\}, \\
U_{P}^{1^{\prime \prime}}= & U_{P}^{1}-\{(1,4)\}=\{(2,1),(2,3),(3,2),(4,1)\} .
\end{aligned}
$$

Hence, continuing as above the fuzzy preference relation $\bar{R}^{1}$ against expert $E_{1}$ is obtained as follows:

$$
\bar{R}^{1}=\left[\begin{array}{cccc}
{[0.5,0.5]} & {[0.5,0.5]} & {[0.6,0.8]} & {[0.3667, .5667]} \\
{[0.4667,0.5222]} & {[0.5,0.5]} & {[0.2889,0.4574]} & {[0.3,0.7]} \\
{[0.2,0.4]} & {[0.3055,0.5389]} & {[0.5,0.5]} & {[0.6,0.9]} \\
{[0.2519,0.4673]} & {[0.3,0.7]} & {[0.1,0.4]} & {[0.5,0.5]}
\end{array}\right]
$$

Step 3: min-consistency preference relation $\widetilde{\widetilde{R}}^{1}$ based on $\bar{R}^{1}$ is obtained as follows:

$$
\widetilde{\bar{R}}^{1}=\left[\begin{array}{cccc}
{[0.5,0.5]} & {[0.4889,0.5166]} & {[0.6,0.8]} & {[0.4497, .6574]} \\
{[0.4834,0.5111]} & {[0.5,0.5]} & {[0.375,0.576]} & {[0.3,0.7]} \\
{[0.2,0.4]} & {[0.424,0.625]} & {[0.5,0.5]} & {[0.6,0.9]} \\
{[0.3426,0.5503]} & {[0.3,0.7]} & {[0.1,0.4]} & {[0.5,0.5]}
\end{array}\right]
$$

Likewise, min-consistency preference relations $\widetilde{\widetilde{R}}^{2}$ and $\widetilde{\widetilde{R}}^{3}$ against the experts $E_{2}$ and $E_{3}$ respec- 
tively, given as below:

$$
\begin{gathered}
\widetilde{\bar{R}}^{2}=\left[\begin{array}{cccc}
{[0.5,0.5]} & {[0.33,0.46]} & {[0.4,0.6]} & {[0.3,0.7]} \\
{[0.54,0.67]} & {[0.5,0.5]} & {[0.7,0.8]} & {[0.46,0.61]} \\
{[0.4,0.6]} & {[0.2,0.3]} & {[0.5,0.5]} & {[0.3,0.4]} \\
{[0.3,0.7]} & {[0.39,0.54]} & {[0.6,0.7]} & {[0.5,0.5]}
\end{array}\right], \\
\widetilde{\bar{R}}^{3}=\left[\begin{array}{cccc}
{[0.5,0.5]} & {[0.4166,0.55]} & {[0.7,0.8]} & {[0.4722,0.599]} \\
{[0.45,0.5834]} & {[0.5,0.5]} & {[0.5333,0.6592]} & {[0.5,0.7]} \\
{[0.2,0.3]} & {[0.3408,0.4667]} & {[0.5,0.5]} & {[0.384,0.4994]} \\
{[0.401,0.5278]} & {[0.3,0.5]} & {[0.5006,0.616]} & {[0.5,0.5]}
\end{array}\right] .
\end{gathered}
$$

Step 4: The collective matrix against all the experts is shown as follows:

$$
\bar{R}^{c}=\left[\begin{array}{cccc}
{[0.5,0.5]} & {[0.4118,0.5089]} & {[0.5667,0.7333]} & {[0.4073,0.6521]} \\
{[0.4911,0.5882]} & {[0.5,0.5]} & {[0.5361,0.6784]} & {[0.42,0.67]} \\
{[0.2667,0.4333]} & {[0.3216,0.4639]} & {[0.5,0.5]} & {[0.428,0.5998]} \\
{[0.3479,0.5927]} & {[0.33,0.58]} & {[0.4002,0.572]} & {[0.5,0.5]}
\end{array}\right]
$$

Step 5: The average degree $\bar{A}_{i}, i=1,2,3,4$, of each alternative is derived by using interval normalizing method given as:

$$
\begin{aligned}
& \bar{A}_{1}=\frac{\sum_{j=1}^{4} \bar{r}_{1 j}^{c}}{\sum_{i=1}^{4} \sum_{j=1}^{4} \bar{r}_{i j}^{c}}=\frac{[1.8858,2.3943]}{[6.9274,9.0726]}=[0.2078,0.3456] ; \\
& \bar{A}_{2}=\frac{\sum_{j=1}^{4} \bar{r}_{2 j}^{c}}{\sum_{i=1}^{n} \sum_{j=1}^{n} \bar{r}_{i j}^{c}}=\frac{[1.9472,2.4366]}{[6.9274,9.0726]}=[0.2146,0.3517] ; \\
& \bar{A}_{3}=\frac{\sum_{j=1}^{4} \bar{r}_{3 j}^{c}}{\sum_{i=1}^{n} \sum_{j=1}^{n} \bar{r}_{i j}^{c}}=\frac{[1.5163,1.997]}{[6.9274,9.0726]}=[0.1671,0.2883] ; \\
& \bar{A}_{4}=\frac{\sum_{j=1}^{4} \bar{r}_{4 j}^{c}}{\sum_{i=1}^{n} \sum_{j=1}^{n} \bar{r}_{i j}^{c}}=\frac{[1.5781,2.2447]}{[6.9274,9.0726]}=[0.1739,0.3240] .
\end{aligned}
$$

Step 6: By using eq. (45), the complementry matrix $D=\left(d_{i j}\right)_{4 \times 4}$ is obtained as follows:

$$
D=\left(d_{i j}\right)_{4 \times 4}=\left[\begin{array}{cccc}
0.5 & 0.4765 & 0.6892 & 0.5964 \\
0.5235 & 0.5 & 0.7147 & 0.619 \\
0.3108 & 0.2853 & 0.5 & 0.4217 \\
0.4036 & 0.381 & 0.5783 & 0.5
\end{array}\right]
$$


Step 7: The ranking value $R_{v}\left(x_{i}\right)$ of alternative $x_{i}, 1 \leq i \leq 4$, is obtained as follows:

$$
\begin{aligned}
& R_{v}\left(x_{1}\right)=\frac{2}{4^{2}} \sum_{j=1}^{4} d_{1 j}=0.2827625 ; \\
& R_{v}\left(x_{2}\right)=\frac{2}{4^{2}} \sum_{j=1}^{4} d_{2 j}=0.29465 ; \\
& R_{v}\left(x_{3}\right)=\frac{2}{4^{2}} \sum_{j=1}^{4} d_{3 j}=0.189725 ; \\
& R_{v}\left(x_{4}\right)=\frac{2}{4^{2}} \sum_{j=1}^{4} d_{4 j}=0.2328625 ;
\end{aligned}
$$

where $\sum_{i=1}^{4} R_{v}\left(x_{i}\right)=1$. Thus, the final ranking of the alternatives is derived as follows:

$$
x_{2}>x_{1}>x_{4}>x_{3} .
$$

Therefore, $x_{2}$ is the best alternative.

The numerical examples show the way to apply the proposed technique to construct the complete IVFPR based on min-consistency. In general, the proposed approach is quite easy for use in estimating unknown preference values.

\section{Conclusion}

In this paper the extended minimum t-norm has been used successfully to determine the missing values in incomplete IVFPR and further extends to construct the min-consistent matrix. Numerical studies show that the proposed technique can handle all type of incomplete IVFPR. Consequently, another algorithm is established to deal with GDM problems with incomplete IVFPRs. This process involves two stages, the estimation of unknown interval-valued preference values and the choice of the best alternative(s).

\section{Bibliography}

[1] S. Alonso, F. Chiclana, F. Herrera, E. Herrera-Viedma, J. Alcala-Fdez, C. Porcel (2008); A Consistency-based Procedure to Estimate Missing Pairwise Preference Values, International Journal of Intelligent Systems, 23 (1): 155-175.

[2] F. Chiclana, F. Herrera, E. Herrera-Viedma (2001); Multiperson Decision Making Based on Multiplicative Preference Relations, European J. of Operational Research, 129: 372385.

[3] F. Chiclana, E. Herrera-Viedma, S. Alonso, F. Herrera; A Note on the Estimation of Missing Pairwise Preference Values: A U-consistency Based Method, International Journal of Uncertainty, Fuzziness and Knowledge-Based Systems, 16: 19-32.

[4] C. Cornelis, G.Deschrijver, E. Kerre (2004); Implication in Intuitionistic Fuzzy and Interval-valued Fuzzy Set Theory: Construction, Classification and Application, International Journal of Approximate Reasoning, 35(1): 55-95.

[5] M. Fedrizzi, G. Silvio (2007); Incomplete Pairwise Comparison and Consistency Optimization, European J. of Operational Research, 183: 303-313. 
[6] J. Fodor, M. Roubens (1994); Fuzzy Preference Modelling and Multicriteria Decision Support, Dordrecht: Kluwer Academic Publishers.

[7] F. Herrera, L. Martíze, P. J. Sánchez (2005); Managing Non-homogeneous Information in Group Decision Making, European Journal of Operational Research, 166: 115-132.

[8] Y. Jiang (2007); An Approach to Group Decision Making Based on Interval Fuzzy Preference Relations, Journal of Systems Science and Systems Engineering, 16(1): 113-120.

[9] E. P. Klement, R. Mesiar, E. Pap (2000); Triangular Norms, Kluwer Academic, Dordrecht.

[10] H. S. Lee, M. T. Chou, H. H. Fang, W. K. Tseng, C. H. Yeh; Estimating Missing Values in Incomplete Additive Fuzzy Preference Relations. In B. Apolloni (Ed.), KES 2007/WIRN 2007, LNAI 4693: $1307-1314$.

[11] L. W. Lee (2012); Group Decision Making with Incomplete Fuzzy Preference Relations Based on the Additive Consistency and the Order Consistency, Expert Systems with Applications, 39: 1166611676 .

[12] R. E. Moore (1979); Methods and Applications of Interval Analysis, SIAM Studies in Applied Mathematics, 2.

[13] S. Orlovsky (1978); Decision-making with a Fuzzy Preference Relation, Fuzzy Sets and Systems, 1: 155167.

[14] T. L. Saaty; The Analytic Hierarchy Process. McGraw-Hill, New York, (1980).

[15] T. Tanino (1984); Fuzzy Preference Orderings in Group Decision Making, Fuzzy Sets and Systems, 12 (2): 117-131.

[16] T. Tanino (1988); Fuzzy Preference Relations in Group Decision Making, in Non-Conventional Preference Relations in Decision Making, eds. J. Kacprzyk and M. Roubens (Springer-Verlag, 1988): 54-71.

[17] Y. M. Wang, Z. P. Fan (2007); Fuzzy Preference Relations: Aggregation and Weight Determination, Computers \& Industrial Engineering, 53: 163-172.

[18] Z. S. Xu (2004); On Compatibility of Interval Fuzzy Preference Relations, Fuzzy Optimization and Decision Making, 3: 217-225.

[19] Z. S. Xu (2004); Goal Programming Models for Obtaining the Priority Vector of Incomplete Fuzzy Preference Relation, International J. of Approximate Reasoning, 36 (3): 261-270.

[20] Z. S. Xu, J. Chen (2008); Some Methods for Deriving the Priority Weights from Interval Fuzzy Preference Relations, European J. of Operational Research, 184: 266-280.

[21] Z. S. Xu, Q. L. Da (2002); The Uncertain OWA Operator, International Journal of Intelligent Systems, 17: 569-575.

[22] Y. J. Xu, Q. L. Da, H. M. Wang (2010); A Note on Group Decision-making Procedure Based on Incomplete Reciprocal Relations, Soft Computing, 15 (7): 1289-1300.

[23] Y. Xu, K. W. Li, H. Wang (2014); Incomplete Interval Fuzzy Preference Relations and their Applications, Computers 83 Industrial Engineering, 67: 93-103.

[24] Y. J. Xu, R. Patnayakuni, H. M. Wang (2013); Logarithmic Least Squares Method to Priority for Group Decision Making with Incomplete Fuzzy Preference Relations, Applied Mathematical Modelling, 37 (4): 2139-2152. 